\title{
Interference effects in LNV and LFV semileptonic decays: the Majorana hypothesis
}

\author{
A. Abada, ${ }^{a}$ C. Hati, ${ }^{b}$ X. Marcano $^{a}$ and A.M. Teixeira ${ }^{b}$ \\ ${ }^{a}$ Laboratoire de Physique Théorique, CNRS, \\ Univ. Paris-Sud, Université Paris-Saclay, 91405 Orsay, France \\ ${ }^{b}$ Laboratoire de Physique de Clermont (UMR 6533), CNRS/IN2P3, \\ Univ. Clermont Auvergne, 4 Av. Blaise Pascal, F-63178 Aubière Cedex, France \\ E-mail: asmaa.abada@th.u-psud.fr, chandan.hati@clermont.in2p3.fr, \\ xabier.marcano@th.u-psud.fr, ana.teixeira@clermont.in2p3.fr
}

Abstract: In the case where the Standard Model is extended by one heavy Majorana fermion, the branching fractions of semileptonic meson decays into same-sign and oppositesign dileptons are expected to be of the same order. As we discuss here, this need not be the case in extensions by at least two sterile fermions, due to the possible destructive and constructive interferences that might arise. Depending on the $C P$ violating phases, one can have an enhancement of the lepton number violating modes and suppression of the lepton number conserving ones (and vice-versa). We explore for the first time the interference effects in semileptonic decays, and illustrate them for a future observation of kaon decays at NA62. We also argue that a non-observation of a given mode need not be interpreted in terms of reduced active-sterile mixings, but that it could instead be understood in terms of interference effects due to the presence of several sterile states; in particular, for differentflavour final state charged leptons, observing a lepton number conserving process and not a lepton number violating one does not rule out that the mediators are Majorana fermions.

Keywords: Beyond Standard Model, CP violation, Kaon Physics, Neutrino Physics

ArXiv EPrint: 1904.05367 


\section{Contents}

1 Introduction 1

2 Semileptonic meson decays with two sterile neutrinos 3

3 Exploring the interference effect 5

4 Illustrative case: semileptonic kaon decays $\quad 8$

5 Conclusions 13

\section{Introduction}

Several extensions of the Standard Model (SM) aiming at explaining oscillation phenomena call upon the introduction of right-handed $(\mathrm{RH})$ neutrinos, and the embedding of the seesaw mechanism onto the SM is one of the most economical mechanisms for the generation of neutrino masses and lepton mixings. The presence of relatively light $\mathrm{RH}$ neutrinos (sterile fermions from the gauge point of view), which have non-negligible mixings with the active ones, leads to the modification of the charged and neutral lepton currents, and thus the new states can give rise to contributions to many observables, ranging from rare transitions and decays at high-intensities to signals at colliders. Mechanisms of neutrino mass generation relying on such sterile fermions are thus particularly appealing, as they offer the possibility of being (albeit indirectly) tested.

The observation of a lepton number violating (LNV) processes signals the existence of New Physics (NP) and is evidence of the Majorana nature of the exchanged fermions. Lepton number violating meson and tau semileptonic decays are examples of such observables; currently, they are the object of extensive world-wide searches, for instance of NA62 (for the light mesons), BES-III for charmed mesons, and LHCb and Belle II for B-mesons and tau leptons.

In this work we address the LNV meson and tau lepton semileptonic three-body decays, $M_{1}^{ \pm} \rightarrow \ell_{\alpha}^{ \pm} \ell_{\beta}^{ \pm} M_{2}^{\mp}$, and $\tau^{ \pm} \rightarrow \ell_{\alpha}^{\mp} M_{1}^{ \pm} M_{2}^{ \pm}$, as well as their lepton number conserving (LNC) counterparts, $M_{1}^{ \pm} \rightarrow \ell_{\alpha}^{ \pm} \ell_{\beta}^{\mp} M_{2}^{ \pm}$, and $\tau^{ \pm} \rightarrow \ell_{\alpha}^{ \pm} M_{1}^{ \pm} M_{2}^{\mp}$ (with the different mesonic states $M_{1,2}$ and charged lepton flavours $\alpha$ and $\beta$ including all kinematically allowed possiblities). When mediated by sterile fermions, the joint study of the LNC and LNV processes allows to shed light on the nature (Dirac or Majorana) of the exchanged mediator.

In the presence of Majorana heavy neutral leptons (a right-handed neutrino, or other sterile fermions), one can have sizeable branching fractions (BRs) for lepton number violating processes, when the Majorana states are produced on-shell [1-11] - the so-called "resonant production" regime. In the case in which a single sterile fermion is present, 
the branching fractions for the semileptonic decays leading to same-sign and opposite-sign dileptons are predicted to be of the same order. Notice that due to the existence of a single heavy fermion, the decay widths are not sensitive to the latter's $C P$ Majorana phases - in particular, one can still have contributions to LNV decays even if all Majorana $C P$ phases are set to zero. Interestingly, should more than one heavy Majorana state be present, the relative size of the LNV and LNC channels may be very different due to interference effects associated with the Dirac and Majorana phases.

In this work, we consider the SM extended by two sterile fermion states which mix with the three active neutrinos; we take the neutrino mass eigenvalues and the lepton mixing matrix to be independent, implying that no assumption is made on the actual mechanism of neutrino mass generation, other than calling upon the presence of additional heavy singlets. This set-up is useful to understand the general phenomenology of a broad class of models where the SM is enlarged only by sterile fermions, such as the type-I seesaw mechanism and its variants. Other than being in the mass range to be produced "on-shell" in the semileptonic decays, the two new states are taken to be sufficiently close in mass to allow for sizeable interference effects (constructive or destructive) in the LNV and LNC decays, in association with the new $C P$-violating phases.

As we will discuss in detail, depending on the sterile fermion parameter space (i.e., their masses, mass difference and mixings to the active neutrinos - including the $C P$ violating phases), the interference effects can lead to various scenarios, ranging from an extreme suppression of the lepton number conserving observables while having large contributions to lepton number violating ones (and this contrary to the usual belief), to the reverse case. Our findings, relying on a complete numerical analysis, strongly suggest that effects leading to suppressions in some channels should be taken into account upon the interpretation of a non-observation of LNV or LNC meson (or tau) semileptonic decays: reduced BRs might result from the interference of (at least) two sterile states with small mass difference(s), and should not be necessarily translated into more stringent bounds on the heavy neutrino couplings to the active ones. More importantly, the observation of a lepton number conserving process accompanied by negative search results for the corresponding LNV modes (with both predicted to be within experimental sensitivity) does not necessarily imply that the mediators are of Dirac nature.

Here we present the results of our study, focusing on the semileptonic kaon decays currently searched for in NA62, that is $K^{ \pm} \rightarrow \ell_{\alpha}^{ \pm} \ell_{\beta}^{ \pm} \pi^{\mp}$ decays and the corresponding lepton number conserving ones, $K^{ \pm} \rightarrow \ell_{\alpha}^{ \pm} \ell_{\beta}^{\mp} \pi^{ \pm}$(where $\alpha, \beta$ denote electrons and/or muons). Kaon decays are used here as an illustrative example, as the results generically hold for all similar semileptonic decays (including tau decays).

Similar studies have focused on the prospects of LNV searches at colliders, see [12], and references therein. Some works have explored the role of the second heavy neutrino concerning the possibility of resonant $C P$ violation [13], in a forward-backward asymmetry at electron-positron collider [14], while others have compared the expected number of events associated with same-sign and opposite-sign dileptons at colliders in the framework of Left- 
Right symmetric models ${ }^{1}[16]$. The latter analysis considered scenarios in which the relative $C P$ violating phases of active-sterile mixings were identical for the heavy neutrinos. In our work - aiming at studying rare meson decays at lower energies - we relax this restrictive hypothesis, which opens the possibility of very distinct behaviours for the LNV and LNC rates, due to the interference effects.

Although the study is carried for a simplified SM extension (the " $3+2$ " minimal model), we emphasise that our results generically apply to complete mechanisms of neutrino mass generation, provided at least two additional states are present, as in the case of low-scale type I seesaw frameworks (in which at least two RH neutrinos are required to accommodate oscillation data).

This work is organised as follows: after an analytical discussion in section 2 of semileptonic meson decay LNC and LNV amplitudes (in the presence of two additional sterile states), we explore in section 3 the interference effects in a generic way, identifying critical regimes and the potential consequences for the relative size of the BRs. Section 4 is dedicated to a full numerical analysis, in which the intereference effects are illustrated for kaon decays. We discuss further points, and summarise our most important findings in the conclusions.

\section{Semileptonic meson decays with two sterile neutrinos}

As stated in the Introduction, we work in the framework of simplified SM extensions via the addition of $N$ extra neutral Majorana fermions, making no assumption on the mechanism of neutrino mass generation (i.e., considering neutrino masses and lepton mixings to be independent). In the presence of new sterile states with non-negligible mixings to the (light) active neutrinos, the leptonic charged current is modified as

$$
-\mathcal{L}_{\mathrm{cc}}=\frac{g}{\sqrt{2}} U_{\alpha i} \bar{\ell}_{\alpha} \gamma^{\mu} P_{L} \nu_{i} W_{\mu}^{-}+\text {H.c. },
$$

in which $i$ denotes the physical neutrino states, from 1 to $3+N$, and $\alpha$ the flavour of the charged leptons. For the case $N=2$ (corresponding to the addition of two states with masses $m_{4,5}$ ), the unitary matrix $U$, which encodes flavour mixing in charged current interactions, can be parametrised in terms of ten rotation matrices and 4 additional phases as follows $[17,18]$

$$
U=R_{45} R_{35} R_{25} R_{15} R_{34} R_{24} R_{14} R_{23} R_{13} R_{12} \operatorname{diag}\left(1, e^{i \varphi_{2}}, e^{i \varphi_{3}}, e^{i \varphi_{4}}, e^{i \varphi_{5}}\right) .
$$

In the above, $R_{i j}$ corresponds to the rotation matrix between the $i$ and $j$ states (each parametrised by a mixing angle $\theta_{i j}$ and a $C P$-violating phase $\left.\delta_{i j}\right)$. For instance, the rotation matrix $R_{14}$ can be explicitly cast as

$$
R_{14}=\left(\begin{array}{ccccc}
\cos \theta_{14} & 0 & 0 & \sin \theta_{14} e^{-i \delta_{14}} & 0 \\
0 & 1 & 0 & 0 & 0 \\
0 & 0 & 1 & 0 & 0 \\
-\sin \theta_{14} e^{i \delta_{14}} & 0 & 0 & \cos \theta_{14} & 0 \\
0 & 0 & 0 & 0 & 1
\end{array}\right)
$$

\footnotetext{
${ }^{1}$ See also the recent work [15].
} 
and similarly for the other $R_{i j}$. We refer to $\delta_{i j}$ and $\varphi_{i}$ respectively as Dirac and Majorana phases since, as we will see later, the $\varphi_{i}$ phases will only appear in LNV processes, while the $\delta_{i j}$ will affect both LNV and LNC processes. Notice that for the considered set-up (adding only SM singlet fermions) not all the mixings and phases are physical and some of them could be removed [19]; for the forthcoming discussion, only the mixings of the sterile states to the active sector are relevant and constitute, therefore, our input parameters. We denote them by

$$
U_{\alpha i}=e^{-i \phi_{\alpha i}}\left|U_{\alpha i}\right|, \quad \alpha=e, \mu, \tau, \text { and } i=4,5 .
$$

Following eq. (2.2), these mixings can be expressed as

$$
\left(\begin{array}{cc}
U_{e 4} & U_{e 5} \\
U_{\mu 4} & U_{\mu 5} \\
U_{\tau 4} & U_{\tau 5}
\end{array}\right) \approx\left(\begin{array}{ll}
s_{14} e^{-i\left(\delta_{41}-\varphi_{4}\right)} & s_{15} e^{-i\left(\delta_{51}-\varphi_{5}\right)} \\
s_{24} e^{-i\left(\delta_{42}-\varphi_{4}\right)} & s_{25} e^{-i\left(\delta_{52}-\varphi_{5}\right)} \\
s_{34} e^{-i\left(\delta_{43}-\varphi_{4}\right)} & s_{35} e^{-i\left(\delta_{53}-\varphi_{5}\right)}
\end{array}\right)
$$

where $s_{i j}=\sin \theta_{i j}$ and where we have neglected terms of $\mathcal{O}\left(s_{i j}^{2}\right)$. For the rest of the analysis, we will nevertheless use the generic notation of eq. (2.4).

We now address the effect of the new mixings on the LNC semileptonic processes $M \rightarrow$ $M^{\prime} \ell_{\alpha}^{ \pm} \ell_{\beta}^{\mp}$ and the corresponding LNV ones $M \rightarrow M^{\prime} \ell_{\alpha}^{ \pm} \ell_{\beta}^{ \pm}, M$ and $M^{\prime}$ being pseudoscalar mesons $^{2}$. Their squared amplitudes (see [11]) are proportional, up to overall constant parameters, to the following:

$$
\begin{aligned}
& \left|\mathcal{A}_{M \rightarrow M^{\prime} \ell_{\alpha}^{+} \ell_{\beta}^{-}}^{\mathrm{LNC}}\right|^{2} \propto\left|U_{\alpha 4} U_{\beta 4}^{*} g\left(m_{4}\right)+U_{\alpha 5} U_{\beta 5}^{*} g\left(m_{5}\right)\right|^{2}, \\
& \left|\mathcal{A}_{M \rightarrow M^{\prime} \ell_{\alpha}^{-} \ell_{\beta}^{+}}^{\mathrm{LNC}}\right|^{2} \propto\left|U_{\alpha 4}^{*} U_{\beta 4} g\left(m_{4}\right)+U_{\alpha 5}^{*} U_{\beta 5} g\left(m_{5}\right)\right|^{2}, \\
& \left|\mathcal{A}_{M \rightarrow M^{\prime} \ell_{\alpha}^{+} \ell_{\beta}^{+}}^{\mathrm{LNV}}\right|^{2} \propto\left|U_{\alpha 4} U_{\beta 4} f\left(m_{4}\right)+U_{\alpha 5} U_{\beta 5} f\left(m_{5}\right)\right|^{2}, \\
& \left|\mathcal{A}_{M \rightarrow M^{\prime} \ell_{\alpha}^{-} \ell_{\beta}^{-}}^{\mathrm{LNV}}\right|^{2} \propto\left|U_{\alpha 4}^{*} U_{\beta 4}^{*} f\left(m_{4}\right)+U_{\alpha 5}^{*} U_{\beta 5}^{*} f\left(m_{5}\right)\right|^{2},
\end{aligned}
$$

leading to

$$
\begin{aligned}
& \left|\mathcal{A}_{M \rightarrow M^{\prime} \ell_{\alpha}^{ \pm} \ell_{\beta}^{\mp}}^{\mathrm{LNC}}\right|^{2} \propto\left|U_{\alpha 4}\right|^{2}\left|U_{\beta 4}\right|^{2}|g(M)|^{2}\left|1+\kappa^{\prime} e^{\mp i\left(\psi_{\alpha}-\psi_{\beta}\right)}\right|^{2}, \\
& \left|\mathcal{A}_{M \rightarrow M^{\prime} \ell_{\alpha}^{ \pm} \ell_{\beta}^{ \pm}}^{\mathrm{LNV}}\right|^{2} \propto\left|U_{\alpha 4}\right|^{2}\left|U_{\beta 4}\right|^{2}|f(M)|^{2}\left|1+\kappa e^{\mp i\left(\psi_{\alpha}+\psi_{\beta}\right)}\right|^{2},
\end{aligned}
$$

where we have defined $\psi_{\alpha} \equiv \phi_{\alpha_{5}}-\phi_{\alpha 4}$, and $M$ is the average mass of the two sterile neutrinos ( $\Delta M$ their mass splitting), so that $m_{4}=M-\Delta M / 2$ and $m_{5}=M+\Delta M / 2$. The functions $f$ and $g$ are the integrals one obtains when computing the decay amplitudes for LNV and LNC semileptonic decays of mesons (details can be found in for instance [11]).

\footnotetext{
${ }^{2}$ Here we present the case of semileptonic meson decays; however, a similar discussion holds for semileptonic tau decays $\tau \rightarrow M M^{\prime} \ell_{\alpha}, \alpha=e, \mu$. Moreover, for simplicity we focus on $M^{+} \rightarrow M^{\prime} \ell_{\alpha}^{+} \ell_{\beta}^{-}$and $M^{+} \rightarrow M^{\prime} \ell_{\alpha}^{+} \ell_{\beta}^{+}$.
} 
The complex quantities $\kappa$ and $\kappa^{\prime}$, which reflect the relative size of the contributions of the two sterile fermions to the processes, are defined as

$$
\kappa \equiv \frac{\left|U_{\alpha 5} U_{\beta 5}\right|}{\left|U_{\alpha 4} U_{\beta 4}\right|} \frac{f\left(m_{5}\right)}{f\left(m_{4}\right)}, \quad \kappa^{\prime} \equiv \frac{\left|U_{\alpha 5} U_{\beta 5}^{*}\right|}{\mid U_{\alpha 4} U_{\beta 4}^{*}} \frac{g\left(m_{5}\right)}{g\left(m_{4}\right)} .
$$

Equations (2.6), (2.7) allow to infer several important points: the LNC decay amplitudes are not sensitive to the $C P$ violating phases $\varphi_{i}$, as these cancel out in the $\psi_{\alpha}-\psi_{\beta}$ combination, therefore revealing their Majorana nature; the LNC decay amplitudes are sensitive to the $\delta_{i j}$ (Dirac) phases, but only in the case of flavour violating final states, i.e. $\alpha \neq \beta$ [13]. On the other hand, the LNV decay amplitudes are sensitive to both Dirac and Majorana $C P$ phases (since the phase appearing in the decay amplitude is the sum of the relative $C P$ phases, $\psi_{\alpha}+\psi_{\beta}$ ), and this even in the case of identical charged leptons in the final state $(\alpha=\beta)$.

In order to discuss the impact of the $C P$ phases on the LNV and LNC decay amplitudes, as well as possible interference effects, we consider the quantity $R_{\ell_{\alpha} \ell_{\beta}}$ defined as

$$
R_{\ell_{\alpha} \ell_{\beta}} \equiv \frac{\Gamma_{M \rightarrow M^{\prime} \ell_{\alpha}^{ \pm} \ell_{\beta}^{ \pm}}^{\mathrm{LNV}}}{\Gamma_{M \rightarrow M^{\prime} \ell_{\alpha}^{ \pm} \ell_{\beta}^{\mp}}^{\mathrm{LNC}}},
$$

and further introduce the ratio $\widetilde{R}_{\ell_{\alpha} \ell_{\beta}}$

$$
\widetilde{R}_{\ell_{\alpha} \ell_{\beta}} \equiv \frac{\Gamma_{M \rightarrow M^{\prime} \ell_{\alpha}^{ \pm} \ell_{\beta}^{\mp}}^{\mathrm{LNC}}-\Gamma_{M \rightarrow M^{\prime} \ell_{\alpha}^{ \pm} \ell_{\beta}^{ \pm}}^{\mathrm{LNV}}}{\Gamma_{M \rightarrow M^{\prime} \ell_{\alpha}^{ \pm} \ell_{\beta}^{\mp}}^{\mathrm{LNC}}+\Gamma_{M \rightarrow M^{\prime} \ell_{\alpha}^{ \pm} \ell_{\beta}^{ \pm}}^{\mathrm{LNV}}}=\frac{1-R_{\ell_{\alpha} \ell_{\beta}}}{1+R_{\ell_{\alpha} \ell_{\beta}}},
$$

with, in both ratios, $\Gamma_{M \rightarrow M^{\prime} \ell_{\alpha}^{ \pm} \ell_{\beta}^{\mp}}^{\mathrm{LNC}} \equiv \Gamma_{M \rightarrow M^{\prime} \ell_{\alpha}^{+} \ell_{\beta}^{-}}^{\mathrm{LNC}}+\Gamma_{M \rightarrow M^{\prime} \ell_{\alpha}^{-} \ell_{\beta}^{+}}^{\mathrm{LNC}}$, in the case in which $\alpha \neq \beta$.

The ratio $R_{\ell_{\alpha} \ell_{\beta}}$ is usually considered to compare LNV to LNC processes (a similar approach to what was done, for instance, in the context of collider searches [16]) and the second ratio, $\widetilde{R}_{\ell_{\alpha} \ell_{\beta}}$, which a priori might seem redundant, will be useful to better understand interference effects.

\section{Exploring the interference effect}

As extensively discussed in [1-11], in addition to being of Majorana nature, the sterile fermions mediating the LNV decays should be produced on-shell, in which case one can have a resonant enhancement of the decay widths. In the narrow-width approximation, this "resonant enhancement" can be understood as an increase of $\mathcal{O}\left(m_{i} / \Gamma_{N_{i}}\right)$ in the decay rates $\left(\Gamma_{N_{i}}\right.$ denoting the width of the heavy sterile state $N_{i}$ ). For this reason, we will assume that the individual widths are very small compared to the sterile neutrino masses, ${ }^{3} \Gamma_{N_{i}} \ll m_{i}$.

In the case of the SM extended by only one heavy Majorana neutrino, we have verified that the predictions for the LNV and LNC decay widths are of the same order, implying

\footnotetext{
${ }^{3}$ Notice that this assumption is well justified, as this is usually the case in seesaw-like models where the sterile neutrinos are lighter than the typical meson masses [20].
} 
that $R_{\ell_{\alpha} \ell_{\beta}}=1$ and thus $\widetilde{R}_{\ell_{\alpha} \ell_{\beta}}=0$. In the presence of two (or more) sterile fermions with (clearly) non-degenerate masses, interference effects are negligible and one recovers the previous predictions for $R$ and $\widetilde{R}$. However, when the mass splitting of the heavy Majorana states is very small, one can have an overlap between their contributions, possibly leading to destructive or constructive interferences. The effect of the overlap will be maximal should the mass splitting be even smaller than the Majorana neutrino decay widths. In turn, this will lead to different predictions for the LNV and LNC decay widths, changing the values $R$ and $\widetilde{R}$. In summary, interference effects are expected to be relevant if both the following conditions are realised:

$$
\Delta M \ll M \quad \text { and } \quad \Delta M<\Gamma_{N},
$$

in which, for simplicity, we have assumed the widths to be the same $\Gamma_{N_{4}}=\Gamma_{N_{5}}=\Gamma_{N}$. With these conditions, and in terms of the $C P$-violating phases, the ratio $R_{\ell_{\alpha} \ell_{\beta}}$ is given as follows

$$
R_{\ell_{\alpha} \ell_{\beta}}=\frac{(1-|\kappa|)^{2}+4|\kappa| \cos ^{2}\left(\frac{\delta \pm\left(\psi_{\alpha}+\psi_{\beta}\right)}{2}\right)}{\left(1-\left|\kappa^{\prime}\right|\right)^{2}+4\left|\kappa^{\prime}\right| \cos ^{2}\left(\frac{\delta^{\prime} \pm\left(\psi_{\alpha}-\psi_{\beta}\right)}{2}\right)}
$$

where we have set $\kappa^{\left({ }^{\prime}\right)}=\left|\kappa^{\left({ }^{\prime}\right)}\right| e^{\left.i \delta^{\prime}\right)}$, and with the \pm referring to the electric charge of the lepton $\alpha$.

Moreover, the coefficients $\kappa$ and $\kappa^{\prime}$ of eq. (2.8) can be expanded as follows

$$
|\kappa| \simeq\left|\kappa^{\prime}\right|=\frac{\left|U_{\alpha 5} U_{\beta 5}^{*}\right|}{\left|U_{\alpha 4} U_{\beta 4}^{*}\right|}\left(1+\mathcal{O}\left(\frac{\Delta M}{\Gamma_{N}}\right)\right) .
$$

In order to have sizeable interference effects, in addition to having a small mass splitting, the relative size of the contributions of the two neutrinos to each amplitude should be of the same order, and not very different from $1,|\kappa| \sim\left|\kappa^{\prime}\right| \approx 1$ (as can be seen from eqs. (2.6), (2.7)), implying that the two neutrinos should mix with similar strength to the relevant active flavours.

Under the hypotheses of eq. (3.1), and in the limit $|\kappa| \sim\left|\kappa^{\prime}\right| \sim 1$, one can derive the ratios $R_{\ell_{\alpha} \ell_{\beta}}$ and $\widetilde{R}_{\ell_{\alpha} \ell_{\beta}}$ in terms of the $C P$-violating phases as

$$
\begin{aligned}
R_{\ell_{\alpha} \ell_{\beta}} & =\frac{\cos ^{2}\left[\frac{1}{2}\left(\psi_{\alpha}+\psi_{\beta}\right)\right]}{\cos ^{2}\left[\frac{1}{2}\left(\psi_{\alpha}-\psi_{\beta}\right)\right]} \\
\widetilde{R}_{\ell_{\alpha} \ell_{\beta}} & =\frac{\sin \psi_{\alpha} \sin \psi_{\beta}}{\cos \psi_{\alpha} \cos \psi_{\beta}+1},
\end{aligned}
$$

where (for simplicity) we have assumed in the last equations that $\delta=\delta^{\prime}=0$. One can immediately notice from eq. (3.4) that, for $\alpha \neq \beta$, the ratio $R_{\ell_{\alpha} \ell_{\beta}}$ can deviate from 1 (larger or smaller) due to the presence of both relative $C P$ violating phases, $\psi_{\alpha}$ and $\psi_{\beta}$.

The effect of the interference between the two sterile fermion contributions can already be seen in the simple limiting case in which the relative $C P$-violating phases are identical $\psi_{\alpha}=\psi_{\beta}$ (the same limit was also used in [16] regarding collider searches). This situation can be realised if, for example, one sets all the Dirac $C P$ phases to zero so that the ratio 


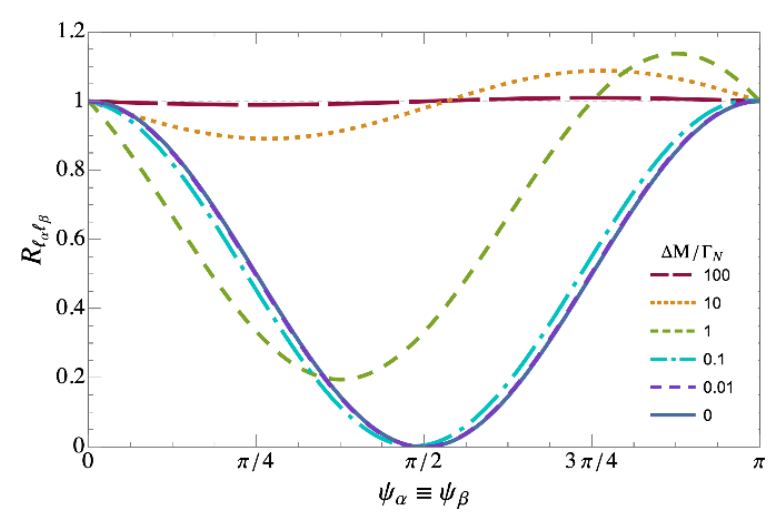

Figure 1. The ratio $R_{\ell_{\alpha} \ell_{\beta}}$, as generically arising from the comparison of the LNC and LNV widths of any given semileptonic meson decay; $R_{\ell_{\alpha} \ell_{\beta}}$ is depicted as a function of a common $C P$ violating phase, $\psi_{\alpha}=\psi_{\beta}$. Several regimes of $\Delta M / \Gamma_{N}$ illustrate how the conditions of eq. (3.1) are crucial to observe maximal interference effects.

$R_{\ell_{\alpha} \ell_{\beta}}$ depends only on the Majorana $C P$ phases. It is important to notice that in such cases (i.e., for $\psi_{\alpha}=\psi_{\beta}$ ) no interference effects (destructive or constructive) occur for the LNC case.

In this limiting case, the ratio $R_{\ell_{\alpha} \ell_{\beta}}$ is illustrated in figure 1 as a function of the common relative $C P$ phase $\left(\psi_{\alpha}=\psi_{\beta}\right)$ for different values of $\Delta M / \Gamma_{N}$. The lines of figure 1 were obtained via a full numerical ${ }^{4}$ evaluation of eq. (2.9), and for the example of $K \rightarrow \pi e \mu$ LNV and LNC decays; here we have set $M=350 \mathrm{MeV}$, and taken an identical strength for the active-sterile mixings, $\left|U_{\alpha 4}\right| \approx\left|U_{\alpha 5}\right|=10^{-5}(\alpha=e, \mu)^{5}$; the sterile neutrino total decay widths are computed following $[4,11,20]$.

Similar analyses were carried for other meson decays and different flavour content of the final lepton states (when kinematically allowed); we have confirmed that in all cases the corresponding $R_{\ell_{\alpha} \ell_{\beta}}$ exhibit an analogous behaviour to that of figure 1. In addition, such behaviour is periodic in the phases $(\bmod \pi)$.

One can also see from figure 1 that the "naïve" limit, $R_{\ell_{\alpha} \ell_{\beta}}=1$, is indeed recovered for the case of vanishing values of the relative phase $\psi$, as expected from eq. (3.4). With the exception of regimes leading to $\Delta M / \Gamma_{N} \gg 1$ - for which there is no visible interference effect and one recovers the expectations of the SM extended by one sterile state (i.e., $R_{\ell_{\alpha} \ell_{\beta}} \simeq 1$ ) - the lines of figure 1 illustrate both constructive and destructive interference effects in the LNV amplitude, clearly signalling the presence of at least two additional sterile states.

The constructive interference effects are typically more important for values of $\psi \sim \pi$; for the curves corresponding to very small $\Delta M / \Gamma_{N} \ll 1$, the constructive interference is still present (provided $\Delta M \neq 0$ ), although not visible due to the resolution of the plot.

\footnotetext{
${ }^{4}$ The numerical analysis was done following the full computation of [11], adapted to the case of two additional sterile states.

${ }^{5}$ Such a choice is inspired by the study of [11], since it leads to contributions to the LNV (and LNC) BRs within future experimental sensitivity, some modes being already in conflict with curent data.
} 
The most remarkable effect in figure 1 is the destructive interference in the LNV amplitude due to the presence of two sterile neutrinos, which coherently interfere: suppressions of order $90 \%$ or above can occur when $\Delta M / \Gamma_{N} \rightarrow 0$. In this situation the interpretation of negative LNV searches does not preclude the observation of a signal for the corresponding LNC channel; especially in the framework of models containing additional Majorana fermions, this might only suggest the presence of at least two states, with non-negligible relative $C P$ violating phases.

Notice that in the case $\psi_{\alpha}=\psi_{\beta}$, our analysis of semileptonic decays - carried in a very generic extension of the SM by two heavy neutral leptons - leads to patterns similar to those of ref. [16] (in which the quantity $R_{\ell \ell}$ denoted the ratio of same-sign to opposite-sign dilepton number of events in hadron colliders).

The constructive/destructive interference effects illustrated in figure 1 are a general feature of semileptonic meson and tau decays. These effects can become even more pronounced if one steps away from the special case where the two relative $C P$ phases are equal (which preserved LNC processes from interferences). In the more general case where $\psi_{\alpha} \neq \psi_{\beta}$, interferences can occur in both LNV and LNC decay amplitudes, leading to enhanced values of $R_{\ell_{\alpha} \ell_{\beta}}$ - or more strikingly, to the suppression of the LNC amplitudes. However, we stress that for LNC processes, interference effects can only occur for different flavour final state charged leptons, i.e. $\ell_{\alpha} \neq \ell_{\beta}$.

Although we do not discuss them here, LNC and LNV four-body semileptonic meson decays (leading to two mesons and two charged leptons in the final state) could also be used to illustrate the interference effects due to the presence of at least two sterile states. We expect that the results for (a generalized definition of) $R_{\ell_{\alpha} \ell_{\beta}}$ would also hold.

\section{Illustrative case: semileptonic kaon decays}

The suppression/enhancement of the LNV and LNC decay amplitudes discussed in the previous section can occur in all meson (and tau) decays for the different (kinematically allowed) final state charged leptons.

An important goal of this study, previously highlighted is the (re-)interpretation of possible deviations of the $R_{\ell_{\alpha} \ell_{\beta}}$ ratio from 1 , under the working hypothesis of the SM extended by at least two sterile fermions. In order to do so, one must be able to single out the heavy Majorana neutrino contributions to both LNV and LNC decays; while LNV transitions are forbidden in the SM, the latter is the source of the dominant contributions concerning LNC semileptonic decays into same-flavoured lepton final states. From here on, we thus focus on LNC different flavour final states $\left(\ell_{\alpha} \neq \ell_{\beta}\right)$, to isolate the sterile neutrino contributions. ${ }^{6}$ Moreover, it is clear that any discussion of $R_{\ell_{\alpha} \ell_{\beta}}$ would ideally require an observation of the branching ratios of both LNV and LNC transitions.

Analyses of (lepton number violating and conserving) semileptonic meson decays and tau-lepton decays have been conducted in the framework of the SM extended by one sterile fermion [1-11], and have allowed to constrain the sterile fermion parameter space. The

\footnotetext{
${ }^{6}$ One could also consider the flavour diagonal channels, and subtract the SM contributions.
} 
recent study of [11], taking into account all available experimental limits, updated the existing bounds for the active-sterile mixings ( $\left|U_{\ell 4}\right|$ and sterile fermion mass); the results of this update suggest that in certain cases, in particular for semileptonic kaon decays, the BRs of the LNV modes $K^{ \pm} \rightarrow \ell_{\alpha}^{ \pm} \ell_{\beta}^{ \pm} \pi^{\mp}(\alpha, \beta=e, \mu)$ and of the SM forbidden LNC mode, $K^{ \pm} \rightarrow e^{ \pm} \mu^{\mp} \pi^{ \pm}$, can both be within the future experimental sensitivity of NA62. For the above reasons, we thus focus on the example of kaon semileptonic LNV and LNC decays to discuss the interference effects. We extend the study of [11] (in particular, concerning the resonant production and the narrow width approximation for the Majorana mediator), and now consider the SM extended by 2 Majorana fermions with masses $m_{i} \in[140,493] \mathrm{MeV}$, in the conditions of eq. (3.1). Our goal is to fully explore the impact of the relative $C P$ violating phases as a source of constructive or destructive interference, leading to deviations of the LNC and LNV decay BRs from what is expected in the most minimal SM extension (via only one sterile state).

The current bounds for the kaon LNV and LNC semileptonic decays are (see, e.g. [21]),

$$
\begin{array}{rlrl}
\operatorname{BR}\left(K^{+} \rightarrow \pi^{+} e^{-} \mu^{+}\right) & \leq 1.3 \times 10^{-11}, & \operatorname{BR}\left(K^{+} \rightarrow \pi^{+} e^{+} \mu^{-}\right) \leq 5.2 \times 10^{-10} \\
\operatorname{BR}\left(K^{+} \rightarrow \pi^{-} e^{+} e^{+}\right) \leq 6.4 \times 10^{-10}, & \operatorname{BR}\left(K^{+} \rightarrow \pi^{-} \mu^{+} \mu^{+}\right) \leq 8.6 \times 10^{-11}, \\
\operatorname{BR}\left(K^{+} \rightarrow \pi^{-} e^{+} \mu^{+}\right) \leq 5.0 \times 10^{-10}, & &
\end{array}
$$

while the future NA62 sensitivity for the processes here discussed is expected to be [22]

$$
\begin{aligned}
\mathrm{BR}\left(K^{+} \rightarrow \pi^{+} e^{ \pm} \mu^{\mp}\right) \leq 0.7 \times 10^{-12}, & \mathrm{BR}\left(K^{+} \rightarrow \pi^{-} e^{+} \mu^{+}\right) \leq 0.7 \times 10^{-12}, \\
\mathrm{BR}\left(K^{+} \rightarrow \pi^{-} e^{+} e^{+}\right) \leq 2 \times 10^{-12}, & \mathrm{BR}\left(K^{+} \rightarrow \pi^{-} \mu^{+} \mu^{+}\right) \leq 0.4 \times 10^{-12} .
\end{aligned}
$$

We begin by displaying in figure 2 the predictions for each of the observables, $\operatorname{BR}\left(K^{+} \rightarrow \pi^{-} e^{+} \mu^{+}\right)$and $\operatorname{BR}\left(K^{+} \rightarrow \pi^{+} e^{ \pm} \mu^{\mp}\right)$, on the parameter space spanned by the two relative phases, $\psi_{e}$ and $\psi_{\mu}$. (We recall that $\psi_{\alpha} \equiv \phi_{\alpha 4}-\phi_{\alpha 5}$, with $\phi_{\alpha i}$ defined in eq. (2.4)). The coloured isosurfaces denote regimes of the corresponding BRs, going from $\mathcal{O}\left(10^{-10}\right)$ (cyan) to nearly 0 (black); the orange lines denote the corresponding curent bounds, see eqs. (4.1), (4.3). Notice that on the right panel, we choose to display only the most conservative of the bounds in eq. (4.1) (to avoid overloading the plot). For the sake of illustration, leading to the numerical results of figure 2, we have taken, $M=350 \mathrm{MeV}$ with $\Delta M \approx 0$ and $\left|U_{\alpha i}\right|=\mathcal{O}\left(10^{-5}\right)(\alpha=e, \mu$ and $i=4,5)$. The associated ratios, $R_{e \mu}$ and $\widetilde{R}_{e \mu}$ (see eqs. (2.9), (2.10)) are displayed in figure 3 , in which the coulored isosurfaces reflect regimes of $\widetilde{R}_{e \mu}\left(R_{e \mu}\right)$ ranging from -1 to 1 ( $\infty$ to 0 ). For completeness, let us notice that the prediction obtained in the case in which the SM is extended by only one sterile fermion (i.e. $N=1$, with $m_{4}=350 \mathrm{MeV}$ and $\left|U_{e 4}\right|=\left|U_{\mu 4}\right| \simeq 10^{-5}$ ), is $\mathrm{BR}=1.8 \times 10^{-10}$ for both LNC and LNV modes, leading to $R_{e \mu}=1$.

Both panels of figure 2 recover and generalize the limiting case $\psi_{\alpha}=\psi_{\beta}$ considered in figure 1 , now for the specific case of $K \rightarrow \pi e \mu$ decays. They also clearly show how the presence of (at least) two sterile states can lead to strong deviations from the minimal extension by a single heavy fermion: for identical values of sterile masses and mixing angles, the variation of the relative phases $\psi_{e}, \psi_{\mu}$ can lead to very different predictions for the BRs. 

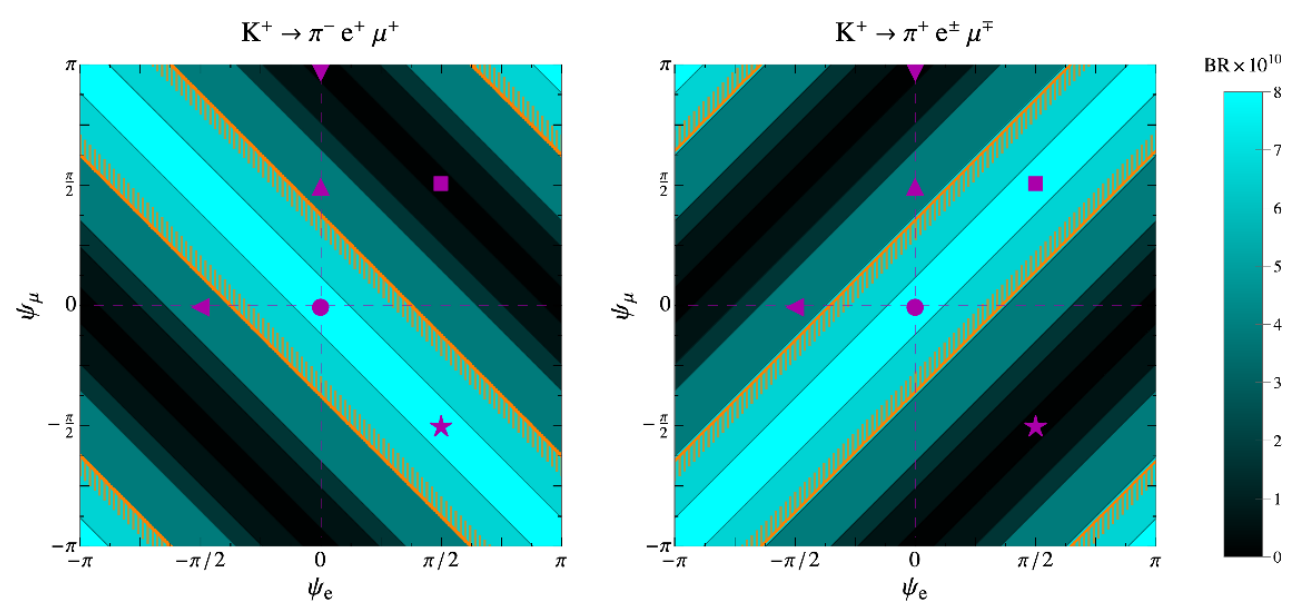

Figure 2. Branching ratios of the LNV $K^{+} \rightarrow \pi^{-} e^{+} \mu^{+}$decay (left) and of the LNC $K^{+} \rightarrow \pi^{+} e^{ \pm} \mu^{\mp}$ decay (right), represented via coloured isosurfaces on the $\psi_{e}-\psi_{\mu}$ plane. In each panel, the orange lines denote the corresponding current experimental bounds (cf. eqs. (4.1), (4.3)). The different superimposed symbols correspond to the illustrative points used in the discussion.

For the left panel, the impact on $\operatorname{BR}\left(K^{+} \rightarrow \pi^{-} e^{+} \mu^{+}\right)$is particularly evident along the direction defined by $\psi_{e}=\psi_{\mu}$, as one can go from BRs already in conflict with experimental bounds for $\psi_{e}=\psi_{\mu}=0$ to a situation of $\operatorname{BR}\left(K^{+} \rightarrow \pi^{-} e^{+} \mu^{+}\right) \approx 0$, due to a maximal destructive interference of the heavy fermion contributions occurring for instance for $\psi_{e}=$ $\psi_{\mu}= \pm \pi / 2$.

Along the $\psi_{e}=\psi_{\mu}$ direction, no effect is manifest for the LNC modes; as seen from eq. (2.6), these depend on the combination $\psi_{\alpha}-\psi_{\beta}$, in which the Majorana phases $\varphi_{4,5}$ cancel out. This can be verified for the LNC $K^{+} \rightarrow \pi^{+} e^{ \pm} \mu^{\mp}$ decays, displayed on the right panel of figure 2 , in which isosurfaces of constant BR are "parallel" to the $\psi_{e}=\psi_{\mu}$ direction. However, any departure from the latter opens the door to interference effects, which are all the more visible for $\psi_{e}=-\psi_{\mu}$, the direction along which the interference effects due to both Majorana and Dirac phases are maximal for the LNC decays. As occurred for the LNV case (left panel), it is visible on the right panel that one goes from $\mathrm{BR}\left(K^{+} \rightarrow \pi^{+} e^{ \pm} \mu^{\mp}\right)$ already excluded by curent data to cases of maximal destructive interference.

Due to the "orthogonal" dependence on the relative phases $\left(\psi_{e} \pm \psi_{\mu}\right.$, respectively for the LNV and LNC decays), the cancellation leading to the extreme case of vanishing LNC amplitudes corresponds in some cases to maximal values for the LNV ones (and vice-versa): specific examples are $\left(\psi_{e}, \psi_{\mu}\right)=(\pi / 2, \pi / 2)$ and $\left(\psi_{e}, \psi_{\mu}\right)=(\pi / 2,-\pi / 2)$, depicted in figure 2 by $\boldsymbol{-}$ and $\star$.

The results of figure 2 clearly illustrate the role of the constructive/destructive interferences regarding the potential observation of each transition, and strongly suggest that any conclusion regarding the contribution of sterile fermions to LNV semileptonic meson decays must be accompanied by the study of the corresponding (flavour violating) LNC mode. Even if a combination of phases leads to an experimentally "blind spot" in which the LNV BR lies beyond sensitivity due to destructive interference effects, the same in- 


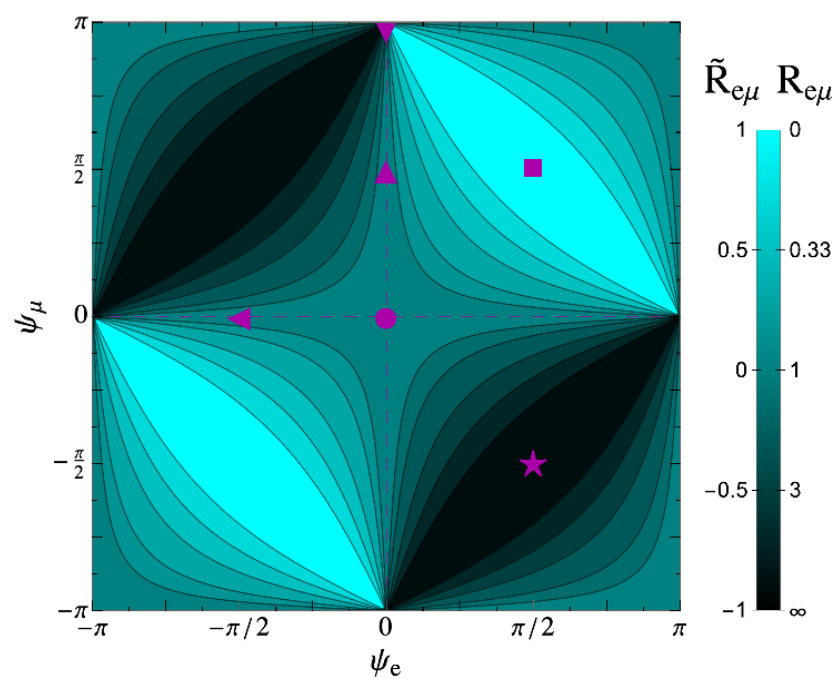

Figure 3. Ratios $R_{e \mu}$ and $\widetilde{R}_{e \mu}$ as defined in eqs. (2.9), (2.10) of the LNV and LNC processes $K \rightarrow \pi e \mu$. The coloured isosurfaces on the $\psi_{e}-\psi_{\mu}$ plane denote regimes for $R_{e \mu}$ and $\widetilde{R}_{e \mu}$. Symbols as in figure 2.

terference might be constructive for the corresponding LNC mode, which could then be associated with a large BR (and vice-versa).

The LNV decay modes leading to same-flavoured final state ${ }^{7}$ leptons (i.e. $\mu^{ \pm} \mu^{ \pm}$and $\left.e^{ \pm} e^{ \pm}\right)$are also sensitive to the relative $C P$ violating phases. The interference for the same flavour final states is maximally constructive (destructive) along the $\psi_{\ell}=0( \pm \pi / 2)$ directions (as can be seen from eq. (2.7)). For instance, some of the regions around $\left(\psi_{e}, \psi_{\mu}\right)=(0, \pm \pi / 2)$ and $( \pm \pi / 2,0)$, that are associated with maximal $/ \mathrm{minimal}$ values of $\operatorname{BR}\left(K^{+} \rightarrow \pi^{-} e^{+} e^{+}\left(\mu^{+} \mu^{+}\right)\right)$, could lead to BRs for the different-flavour final states (both LNV and LNC) well beyond experimental sensitivity (and conversely).

The above discussion and the inferred conclusions become even more straightforward when cast in terms of the ratios $R_{e \mu}$ and $\widetilde{R}_{e \mu}$, as shown in figure 3. Let us then consider several possible experimental scenarios, for instance in association with a future measurement of the LNV and/or LNC kaon decays by NA62 (see eqs. (4.4) for the future experimental sensitivity).

If (within experimental precision) both measurements are approximately compatible with $R_{e \mu} \sim 1$, then interpreting an observation in terms of processes mediated by an onshell sterile neutrino either calls upon scenarios with a single state, or then extensions via several states, either with sizeable mass differences, or then close in mass but with very specific configurations of the $C P$ phases (all cases leading to $R_{e \mu}=1$ and consequently to $\left.\widetilde{R}_{e \mu}=0\right)$.

If the NA62 results translate into a ratio $R_{e \mu}<1$, then an interpretation in terms of a SM extension via sterile states would imply that one is in the presence of at least two additional fermions, both with masses within the region allowing for on-shell production

\footnotetext{
${ }^{7}$ We do not show the numerical results for these channels, although we have verified that they also follow eq. (2.7).
} 
(approximately between $\sim 0.1 \mathrm{GeV}$ and $0.5 \mathrm{GeV}$ ); more importantly it clearly suggests that the sterile fermions are on the regime leading to significant interference effects (see eq. (3.1)). Both measurements, i.e. $K^{+} \rightarrow \pi^{-} e^{+} \mu^{+}$and $K^{+} \rightarrow \pi^{+} e^{-} \mu^{+}$could then be translated into corresponding regions in the $\psi_{e}-\psi_{\mu}$ plane. It is worth emphasising that, as can be inferred from eqs. (4.1)-(4.4), measurements can in principle lead to $R_{e \mu}$ as small as $\sim 10^{-2}$. As already suggested from the general discussion of section 3 (cf. figure 1 , in which the limiting case $\psi_{e}=\psi_{\mu}$ had been considered), such sizeable deviations from $R_{e \mu} \sim 1$ strongly point towards a scenario of $\Delta M / \Gamma_{N} \ll 1$, and to very strong destructive interference effects in the LNV amplitude. This corresponds to $\psi_{e} \sim \psi_{\mu} \sim \pm \pi / 2$, as can be verified in figure 3 .

A possible measurement of the LNV same-flavoured lepton final state BRs would then be highly complementary, and would allow to put our working hypothesis to the test (i.e. the presence of at least two Majorana fermions, with very close masses and non-vanishing relative $C P$ phases). If the two additional observations ${ }^{8}$ are found to be compatible with the model's predictions (for the hinted regimes of the phases), this would naturally strenghten the underlying assumptions. The symbols $\boldsymbol{\Delta}$ and $\boldsymbol{\triangleleft}$ respectively corresponding to $\left(\psi_{e}, \psi_{\mu}\right) \approx$ $(0, \pi / 2)$ and $\left(\psi_{e}, \psi_{\mu}\right) \approx(-\pi / 2,0)$, illustrate this: partial destructive interferences in $K^{+} \rightarrow$ $\pi^{\mp} e^{+} \mu^{ \pm}$would lead to $R_{e \mu} \sim 1$, potentially observable $K^{+} \rightarrow \pi^{-} e^{+} e^{+}\left(\mu^{+} \mu^{+}\right)$, but a maximal destructive interference in $K^{+} \rightarrow \pi^{-} \mu^{+} \mu^{+}\left(e^{+} e^{+}\right)$- which would then likely lie beyond experimental reach.

If neither LNC and LNV modes are observed, the role of the LNV decays into sameflavoured final state leptons is also very important: a possible measurement at NA62 could still point towards a SM extension via at least two sterile neutrinos, whose relative phases account for a full destructive interference in the different-flavour final states, and for a constructive one in the same-flavoured LNV decay modes. For instance, this could occur for $\left(\psi_{e}, \psi_{\mu}\right) \approx(0, \pi)$, denoted by $\mathbf{\nabla}$ on figures 2 and 3 .

Likewise, following the same reasoning as above, one can also have $R_{e \mu}>1$, which would be a consequence of an important destructive interference on the LNC modes.

All the discussion here held in terms of $R_{e \mu}$ can also be translated in terms of $\widetilde{R}_{e \mu}$ (defined in eq. (2.10)), which has the advantage of lying in the range $[-1,1]$, depending on the effect of the interferences. Figure 3 summarises the information conveyed by both panels of figure 2, and suggests how an experimental observation of the LNV and/or LNC channels, if interpreted under the current hypothesis, could help determining the relative $C P$ phases of the sterile fermions.

Although figure 3 corresponds to an analysis of the kaon sector, given that interference effects on any meson semileptonic LNV and LNC decay lead to the same behaviour in terms of the ratios $\widetilde{R}_{\alpha \beta}$ or $R_{\alpha \beta}$ (for $\alpha \neq \beta$ ), it is important to notice that figure 3 in fact illustrates in a generic way the impact of the $C P$ phases that might be present in extensions of the SM by at least two sterile fermions.

Finally, we emphasise that a crucial outcome of our analysis is that a non-observation of the LNV (or LNC) mode should not be directly translated into more stringent bounds

\footnotetext{
${ }^{8}$ The observation of the $K^{+} \rightarrow \pi^{-} \mu^{+} \mu^{+}$, if interpreted under the current hypotheses, would further constrain the masses of the sterile neutrinos, $m_{i} \in \sim[250,390] \mathrm{MeV}$, see [11].
} 
for the sterile mixing angles; it could instead suggest that more than one sterile fermion is present, and interference effects could lead to deviations from the case of a single state. A particularly important consequence concerns finals states with different flavoured charged leptons: if only an LNC mode is observed (even if the associated LNV rate is expected to be within experimental reach), this does not imply that the heavy mediator is a Dirac particle; on the contrary, the processes can be mediated by two Majorana states whose relative phases are at the origin of a maximally destructive interference in the LNV modes (we refer to the case illustrated by on figures 2 and 3. (An extreme case would correspond to having two nearly degenerate Majorana states combining to form a pseudo-Dirac fermion leading to cancellation of all LNV channels, and thus to $R_{\alpha \beta}=0$, as occurs in low scale seesaw mechanisms with approximate lepton number conservation.)

\section{Conclusions}

In this work we have studied for the first time the impact of constructive and destructive interference effects on the contributions of sterile Majorana fermions (such as $\mathrm{RH}$ neutrinos) to the decay rates of lepton number conserving and lepton number violating semileptonic decays.

Previous studies of LNV meson decays have focused on the resonant enhancement of the corresponding widths due to the presence of an on-shell Majorana fermion; the additional sterile state can also mediate LNC semileptonic decays; the decay rates into same-sign and opposite-sign dileptons are expected to be of the same order (i.e., the ratio $\left.R_{\ell_{\alpha} \ell_{\beta}}=1\right)$.

We have extended these studies, now taking into account the presence of at least two sterile neutrinos (with masses allowing for on-shell production), and associated $C P$ violating Dirac and Majorana phases. Provided the mass difference of the new states is small enough $\left(\Delta M / \Gamma_{N} \leq 1\right)$, we have shown that the $C P$ violating phases can lead to constructive/destructive interference effects in the BRs of the decays, reflected in deviations from $R_{\alpha \beta}=1$. We also highlighted that a non-observation of the LNV (or LNC) mode should not be directly translated into more stringent bounds for the sterile mixing angles; it could instead suggest that more than one state is present. Furthermore, observing only lepton number conserving modes (and no LNV transitions) does not rule out that the mediators are indeed of Majorana nature.

Our analysis strongly motivates a re-interpreation of the curent negative search results for LNV and LNC semileptonic meson decays in terms of more than one sterile neutrino; this calls upon a thorough study in terms of an enlarged parameter space. This is the object of an ongoing work [23].

We have illustrated the interference effects for several semileptonic kaon decays: the LNV $K^{ \pm} \rightarrow \ell_{\alpha}^{ \pm} \ell_{\beta}^{ \pm} \pi^{\mp}(\alpha, \beta=e$ and/or $\mu)$, and the corresponding lepton number conserving processes, $K^{ \pm} \rightarrow \ell_{\alpha}^{ \pm} \ell_{\beta}^{\mp} \pi^{ \pm}(\alpha \neq \beta)$, all presently searched for in NA62. We have discussed the possible $R_{e \mu}$ scenarios arising from a future observation of the corresponding decays, and emphasised the relevance of taking into account all four decay modes. Not only this allows to refine the information on the relative phases, but offers independent means to probe the underlying SM extension via sterile fermions. 
A future observation of such processes - if interpreted under the proposed paradigm of at least two sterile fermions - opens a unique window onto the heavy sector parameter space: firstly their average sterile mass is constrained to lie on the resonant production interval; secondly, one can identify ranges of variation for the different couplings (in the case of kaon decays, $\left|U_{e i}\right|$ and $\left.\left|U_{\mu i}\right|\right)$; finally, information can also be inferred on the relative $C P$ violating phases (even if not disentangling the Dirac from the Majorana ones). This information can be ultimately used to probe if the heavy Majorana fermions responsible for the observed LNV and LNC transitions are an integral part of a given mechanism of neutrino mass generation.

\section{Acknowledgments}

We acknowledge support within the framework of the European Union's Horizon 2020 research and innovation programme under the Marie Sklodowska-Curie grant agreements No 690575 and No 674896.

Open Access. This article is distributed under the terms of the Creative Commons Attribution License (CC-BY 4.0), which permits any use, distribution and reproduction in any medium, provided the original author(s) and source are credited.

\section{References}

[1] M. Flanz, W. Rodejohann and K. Zuber, Bounds on effective Majorana neutrino masses at HERA, Phys. Lett. B 473 (2000) 324 [Erratum ibid. B 480 (2000) 418] [hep-ph/9911298] [INSPIRE].

[2] K. Zuber, New limits on effective Majorana neutrino masses from rare kaon decays, Phys. Lett. B 479 (2000) 33 [hep-ph/0003160] [INSPIRE].

[3] A. Atre, V. Barger and T. Han, Upper bounds on lepton-number violating processes, Phys. Rev. D 71 (2005) 113014 [hep-ph/0502163] [INSPIRE].

[4] A. Atre, T. Han, S. Pascoli and B. Zhang, The search for heavy Majorana neutrinos, JHEP 05 (2009) 030 [arXiv: 0901.3589] [INSPIRE].

[5] W. Rodejohann, Neutrino-less double beta decay and particle physics, Int. J. Mod. Phys. E 20 (2011) 1833 [arXiv:1106.1334] [INSPIRE].

[6] J.C. Helo, S. Kovalenko and I. Schmidt, On sterile neutrino mixing with $\nu_{\tau}$, Phys. Rev. D 84 (2011) 053008 [arXiv: 1105.3019] [INSPIRE].

[7] J.-H. Liu, J. Zhang and S. Zhou, Majorana neutrino masses from neutrinoless double-beta decays and lepton-number-violating meson decays, Phys. Lett. B 760 (2016) 571 [arXiv: 1606. 04886] [INSPIRE].

[8] N. Quintero, Constraints on lepton number violating short-range interactions from $|\Delta L|=2$ processes, Phys. Lett. B 764 (2017) 60 [arXiv:1606.03477] [INSPIRE].

[9] G. Moreno and J. Zamora-Saa, Rare meson decays with three pairs of quasi-degenerate heavy neutrinos, Phys. Rev. D 94 (2016) 093005 [arXiv:1606.08820] [INSPIRE]. 
[10] J. Zamora-Saa, Resonant CP violation in rare $\tau^{ \pm}$decays, JHEP 05 (2017) 110 [arXiv: 1612.07656] [INSPIRE].

[11] A. Abada et al., Effective Majorana mass matrix from tau and pseudoscalar meson lepton number violating decays, JHEP 02 (2018) 169 [arXiv:1712.03984] [INSPIRE].

[12] Y. Cai, T. Han, T. Li and R. Ruiz, Lepton number violation: seesaw models and their collider tests, Front. in Phys. 6 (2018) 40 [arXiv:1711.02180] [INSPIRE].

[13] S. Bray, J.S. Lee and A. Pilaftsis, Resonant CP-violation due to heavy neutrinos at the LHC, Nucl. Phys. B 786 (2007) 95 [hep-ph/0702294] [INSPIRE].

[14] P. Hernández, J. Jones-Pérez and O. Suarez-Navarro, Majorana vs. Pseudo-Dirac neutrinos at the ILC, Eur. Phys. J. C 79 (2019) 220 [arXiv:1810.07210] [InSPIRE].

[15] P.S. Bhupal Dev, R.N. Mohapatra and Y. Zhang, Probing heavy neutrino mixing and associated CP-violation at future hadron colliders, arXiv:1904.04787 [INSPIRE].

[16] A. Das, P.S.B. Dev and R.N. Mohapatra, Same sign versus opposite sign dileptons as a probe of low scale seesaw mechanisms, Phys. Rev. D 97 (2018) 015018 [arXiv:1709.06553] [INSPIRE].

[17] A. Abada and T. Toma, Electric dipole moments of charged leptons with sterile fermions, JHEP 02 (2016) 174 [arXiv: 1511.03265] [INSPIRE].

[18] A. Abada, A. Hernández-Cabezudo and X. Marcano, Beta and neutrinoless double beta decays with KeV sterile fermions, JHEP 01 (2019) 041 [arXiv: 1807.01331] [INSPIRE].

[19] J. Schechter and J.W.F. Valle, Neutrino masses in $\mathrm{SU}(2) \times \mathrm{U}(1)$ theories, Phys. Rev. D 22 (1980) 2227 [INSPIRE].

[20] K. Bondarenko, A. Boyarsky, D. Gorbunov and O. Ruchayskiy, Phenomenology of GeV-scale heavy neutral leptons, JHEP 11 (2018) 032 [arXiv:1805.08567] [INSPIRE].

[21] Particle Data Group collaboration, Review of particle physics, Phys. Rev. D 98 (2018) 030001 [INSPIRE].

[22] A. Ceccucci, Kaons at CERN: the NA62 experimental program, PoS (CD15) 006.

[23] A. Abada, C. Hati, X. Marcano and A.M. Teixeira, to appear. 